\section{Cureus}

\title{
Automated Whole Brain Tractography Affects Preoperative Surgical Decision Making
}

Hesham Zakaria ${ }^{1}$, Sameah Haider ${ }^{2}$, Ian Lee ${ }^{3}$

1. Department of Neurological Surgery, Henry Ford Health System 2. Neurological Surgery, Henry Ford Hospital, Detroit, USA 3. Neurosurgery, Henry Ford Hospital, Detroit, USA

$\square$ Corresponding author: Hesham Zakaria, hzakari1@hfhs.org

Disclosures can be found in Additional Information at the end of the article

\section{Abstract}

Surgery in and around eloquent brain structures poses a technical challenge when the goal of surgery is maximal safe resection. Magnetic resonance imaging (MRI) has revolutionized the diagnosis and treatment of neurological disorders, but tractography still remains limited in terms of utility because of the requisite manual labor and time required combined with the high risk of bias and inaccuracy. Automated whole brain tractography (AWBT) has simplified this workflow, overcoming historical barriers, and allowing for integration into modern neuronavigation. However, current literature showing the usefulness of this new technology is limited. In this study, we aimed to illustrate the utility of AWBT during cranial surgery and its ability to affect presurgical and intraoperative clinical decision making. We performed a retrospective chart review of cases that underwent AWBT for one year from July 2016 to July 2017. All patients underwent conventional anatomic MRI with and without contrast sequences, in addition to diffusion tensor imaging (DTI) on a 3 Tesla MRI scanner (Ingenia 3.0T, Philips, Amsterdam NL). Post-hoc AWBT processing was performed on a separate workstation. Patients were subsequently grouped into those that had undergone either language or motor mapping and those that did not. We compared both sets of patients to see any differences in patient age, sex, laterality of surgery, depth of resection from cortical surface, and smallest distance between the lesion and adjacent eloquent white matter tracts. We identified illustrative cases which demonstrated the ability of AWBT to affect surgical decision making. In this singlecenter series, we identified 73 total patients who underwent AWBT for intracranial surgery, of which 28 patients underwent either speech or language mapping. When comparing mapping to non-mapping patients, we found no difference with respect to age, gender, laterality of surgery, or whether the surgery was a revision. The distance between the lesion and eloquent white matter tracts demonstrated a statistically significant difference between mapping and non-mapping patients, namely in the corticospinal tract $(\mathrm{p}<0.0001)$, the superior longitudinal fasciculus ( $p<0.0001)$, and the arcuate fasciculus $(p<0.004)$. Patients who underwent mapping were at equal risk for having a postoperative deficit $(p=0.772)$ but had an improved chance of recovery $(p=0.041)$ after surgery. We believe this phenomenon is related to increased awareness and avoidance of functional tissue during surgery, which occurs due to the combination of preoperatively identifying white matter tracts with AWBT and intraoperatively testing margins with mapping. We provide two illustrative cases that show the impact of AWBT on patient outcomes. In conclusion, AWBT is relatively simple to perform and provides vital information for surgeons about eloquent white matter tracts that can be used to help improve patient outcomes.

Categories: Radiology, Neurosurgery, Quality Improvement

Keywords: neurosurgical outcomes, automated whole brain tractography, neurosurgery, neuro- 
oncology, tractography, brain mri, glioblastoma, neuronavigation

\section{Introduction}

Techniques for identifying white matter tracts have existed for decades [1]; however, traditional (ie, manual) tractography has several limitations which hinder its routine application. With manual tractography, a fiber tract is localized first by selecting specific regions of interest (ROI) that are thought to be along the course of the intended white matter tract. These ROIs are defined by anatomical reference points, and it is from these regions that white matter fiber tracts are derived. Consequently, manual tractography is limited by its prolonged workflow, user bias, and anatomical distortions which may further compound human error [2]. This is problematic because the applicability of tractography for a neurosurgeon is to help identify essential white matter tracts that may be around a potentially resectable lesion, such as a tumor, which by its very nature will have distorted anatomy [3]. Given these limitations, white matter tractography has been utilized infrequently and with skepticism in general practice.

New technologies have recently emerged which seem to have overcome the intrinsic limitations of manual tractography. Advancements in automating tract-based analysis of diffusion tensor imaging (DTI) obtained with magnetic resonance imaging (MRI) have dramatically cut the labor and time required for tractography acquisition, as well as improved reliability and validity [4]. With the automated algorithm, diffusion tensor data from each voxel of a brain MRI are combined to extrapolate the major tract bundles--thereby identifying the theoretical end of fiber tracts by localizing the points of lowest anisotropy. Ultimately, this generates an accurate picture of major white matter tracts in the brain, an approach known formally as automated whole brain tractography (AWBT). AWBT stands in stark contrast to manual tractography, which is labor intensive, intrinsically bias prone, and can only capture one tract at a time between predetermined ROIs [5].

AWBT has the potential to garner widespread use with its ease of implementation and intuitive integration with stereotactic neuronavigation. We describe herein our case series of patients in which AWBT augmented our presurgical planning as well as intraoperative extent of resection. In addition, we also present two illustrative cases where AWBT was foundational to presurgical planning, surgical decisions, and ultimately patient outcomes.

\section{Case Presentation}

Under a previously approved institutional review board (IRB \#9755) study designed to examine the outcomes in craniotomies for tumor resection, we retrospectively reviewed the most recent patients in whom AWBT was utilized. We identified 73 consecutive patients that underwent open surgical resection of an intracranial tumor using the Synaptive Brightmatter ${ }^{\mathrm{TM}}$ System (Synaptive Medical, Toronto, Canada) between July 2016 to July 2017. All patients underwent conventional anatomic MRI with and without contrast sequences in addition to DTI on a 3 Tesla MRI scanner (Ingenia 3.0T, Philips, Amsterdam NL). Post-hoc AWBT processing was performed on a separate PC workstation using BrightMatter software (Synaptive Medical, Toronto Canada). Final patient registration to the navigation system occurred immediately preoperatively and was confirmed again during surgery using anatomic landmarks. We divided patients into those that had undergone mapping (either language or motor) and those that did not. In both sets of patients, we reported patient age at the time of surgery, gender, specific procedure performed, laterality of the procedure, depth of resection from cortical surface, and the smallest distance between the lesion and directly adjacent eloquent white matter tracts. All mapping surgeries were performed by two board certified neurosurgeons who specialize in oncologic surgery and are experienced in mapping. In our institution, we routinely perform tractography for lesions directly abutting major white matter tracts (i.e., the corticospinal tract, the superior longitudinal fasciculus, and the arcuate fasciculus). Student's unpaired t-test and 


\section{Cureus}

Fisher's exact test were used to compare variables when appropriate. Outcomes, including temporary and permanent neurological outcomes, were also reported based on in-depth chart review of individual hospital courses. A temporary deficit was defined as a change in the neurological examination that returned to preoperative baseline within three months. Any persistent changes from baseline, whether anticipated or unanticipated, were considered permanent.

\section{Results}

Of the 73 total patients, 28 underwent either speech or motor mapping with AWBT (Table 1) and 45 utilized AWBT alone (Table 2). Specific demographics and comparison statistics between each group can be found in Table 3. When comparing the two groups of patients, there was no statistically significant difference in age, gender, laterality of surgery, and whether the surgery was a revision. Patients that underwent mapping did not have lesions of different depths; however, they did have lesions that were significantly closer to specific white matter tracts, namely the corticospinal [ $p<0.0001]$, superior longitudinal fasciculus [p $<0.0001]$, or arcuate fasciculus $[\mathrm{p}=0.004]$. This makes sense intuitively, as patients who require supplementary mapping are thought to have higher risk lesions that are in close proximity to eloquent areas, such as white matter tracts, and therefore benefit from the additional protection of mapping. While there was no statistical difference in neurological deficit after surgery between mapping and non-mapping cases $(p=0.772)$, patients who underwent mapping had a higher rate of postoperative recovery $(\mathrm{p}=0.041)$. This finding is crucial as, despite the notion that these patients have higher risk surgeries, they are at no increased risk for postoperative deficits and are more likely to recover after a deficit. This further signifies that healthy perilesional tissue was not irreversibly damaged.

\begin{tabular}{|c|c|c|c|c|c|c|c|c|c|c|c|c|c|c|c|}
\hline $\mathrm{Pt}$ & Age & Sex & Side & Craniotomy & $\begin{array}{l}\text { Revision } \\
\text { Surgery }\end{array}$ & $\begin{array}{l}\text { Speech } \\
\text { Map }\end{array}$ & $\begin{array}{l}\text { Motor } \\
\text { Map }\end{array}$ & $\begin{array}{l}\text { Depth of } \\
\text { Resection } \\
\text { (MM) }\end{array}$ & $\begin{array}{l}\text { MM } \\
\text { From } \\
\text { CST }\end{array}$ & $\begin{array}{l}\text { MM } \\
\text { from } \\
\text { SLF }\end{array}$ & $\begin{array}{l}\text { MM } \\
\text { from } \\
\text { AF }\end{array}$ & $\begin{array}{l}\text { New } \\
\text { Postop } \\
\text { Deficit }\end{array}$ & $\begin{array}{l}\text { Deficit Recovery } \\
\text { within } 3 \mathrm{mo} \text { ? }\end{array}$ & Histology & $\begin{array}{l}\text { Major } \\
\text { Morbidity }\end{array}$ \\
\hline 1 & 75 & M & Left & Parietal & No & Yes & No & 31 & 19.6 & 2 & 2 & No & & Glioblastoma & None \\
\hline 2 & 70 & M & Right & Frontal & Yes & Yes & Yes & 14.2 & 0 & 17.1 & 22.1 & No & & $\begin{array}{l}\text { Metastatic Squamous } \\
\text { Cell }\end{array}$ & None \\
\hline 3 & 45 & $\mathrm{~F}$ & Right & $\begin{array}{l}\text { Fronto- } \\
\text { parietal }\end{array}$ & Yes & No & Yes & 32.7 & 0 & 11.3 & 13.2 & Yes & Yes & $\begin{array}{l}\text { Metastatic Poorly } \\
\text { Differentiated } \\
\text { Carcinoma }\end{array}$ & None \\
\hline 4 & 31 & $\mathrm{~F}$ & Right & Frontal & Yes & No & Yes & 47.4 & 28.6 & 24.5 & 30.2 & No & & Anaplastic Astrocytoma & None \\
\hline 5 & 26 & M & Right & Parietal & No & No & Yes & 54.5 & 0 & 13 & 15 & No & & Glioblastoma & None \\
\hline 6 & 29 & M & Right & Frontal & Yes & Yes & Yes & 37.5 & 12.3 & 17.3 & 8.6 & No & & Oligo & None \\
\hline 7 & 26 & M & Left & $\begin{array}{l}\text { Fronto- } \\
\text { temporal }\end{array}$ & No & Yes & Yes & 40.6 & 0 & 0 & 0 & Yes & No & Glioblastoma & None \\
\hline 8 & 36 & M & Left & Frontal & Yes & Yes & Yes & 60.5 & 8 & 0 & 5.3 & No & & Glioblastoma & None \\
\hline 9 & 53 & $\mathrm{~F}$ & Left & Temporal & Yes & Yes & No & 28 & 24.3 & 0 & 0 & Yes & Yes & Anaplastic Oligo & None \\
\hline 10 & 38 & $\mathrm{~F}$ & Right & Parietal & Yes & No & Yes & 24.5 & 16.7 & 23.7 & 18.5 & No & & Oligo & None \\
\hline 11 & 67 & $F$ & Left & Parietal & No & No & Yes & 68.3 & 5 & 0 & 0 & No & & Glioblastoma & None \\
\hline
\end{tabular}




\section{Cureus}

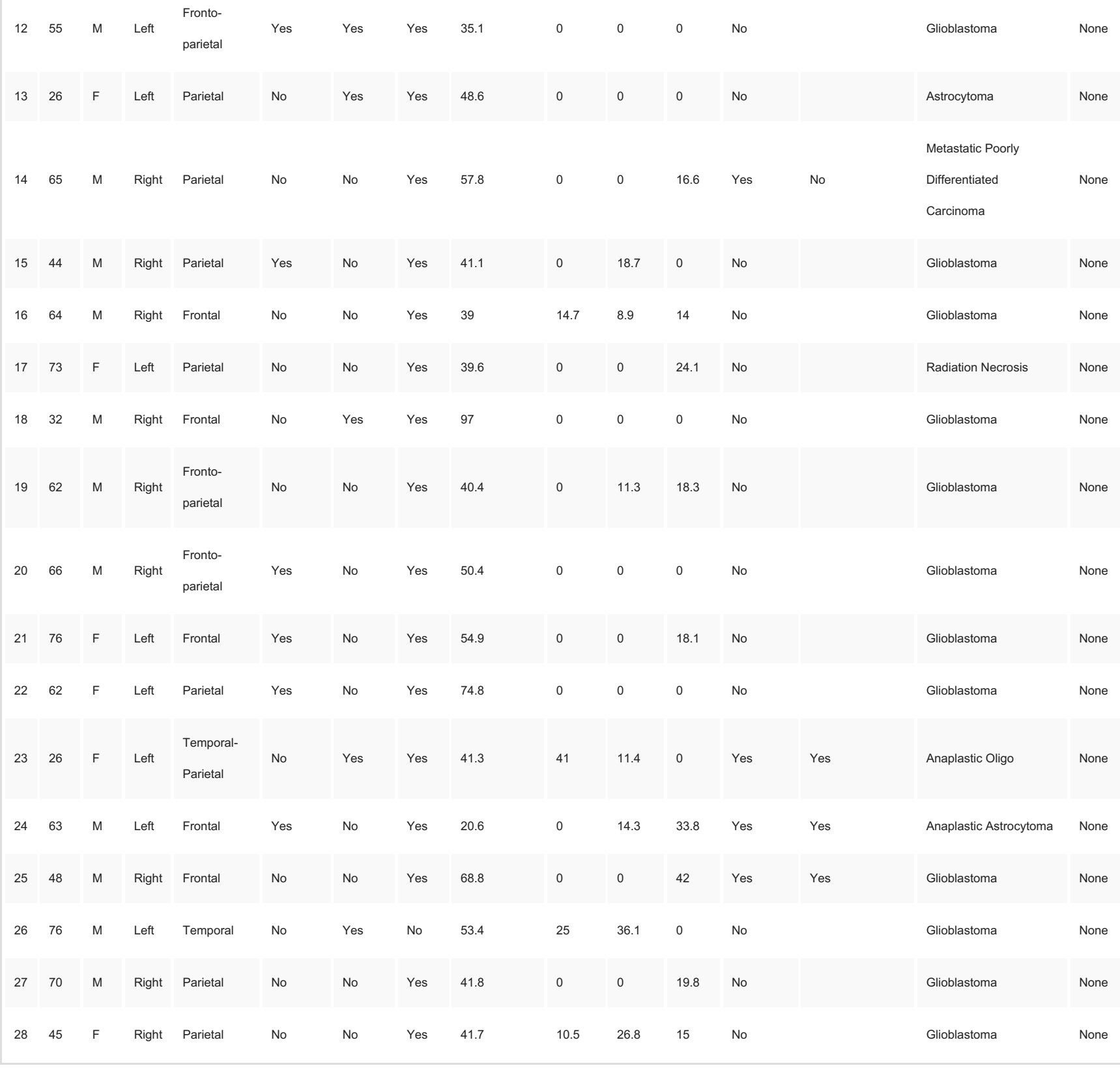

\section{TABLE 1: Patients Who Underwent Speech and/or Motor Mapping}

WHO: World Health Organization

CST: Corticospinal Tract

SLF: Superior Longitudinal Fasciculus

AF: Arcuate Fasciculus

Oligo: Oligodendroglioma

MM: Millimeters

Pt: Patient 


\section{Cureus}

\begin{tabular}{|c|c|c|c|c|c|c|c|c|c|c|c|c|c|}
\hline $\begin{array}{l}\mathbf{P t} \\
\#\end{array}$ & Age & Sex & Side & Craniotomy & $\begin{array}{l}\text { Revision } \\
\text { Surgery }\end{array}$ & $\begin{array}{l}\text { Depth of } \\
\text { Resection } \\
\text { (MM) }\end{array}$ & $\begin{array}{l}\text { MM } \\
\text { From } \\
\text { CST }\end{array}$ & $\begin{array}{l}\text { MM } \\
\text { from } \\
\text { SLF }\end{array}$ & $\begin{array}{l}\mathrm{MM} \\
\text { from } \\
\mathrm{AF}\end{array}$ & $\begin{array}{l}\text { New } \\
\text { Postop } \\
\text { Deficit }\end{array}$ & $\begin{array}{l}\text { Deficit } \\
\text { Recovery } \\
\text { within } 3 \mathrm{mo} \text { ? }\end{array}$ & Histology & $\begin{array}{l}\text { Major } \\
\text { Morbidity }\end{array}$ \\
\hline 1 & 55 & M & Left & Parietal & Yes & 12.2 & 30 & 15 & 15 & No & & $\begin{array}{l}\text { Metastatic Squamous } \\
\text { Cell }\end{array}$ & \\
\hline 2 & 45 & M & Right & Parietal & No & 76.4 & 36 & 0 & 0 & Yes & No & Glioblastoma & $\begin{array}{l}\text { Intracerebral } \\
\text { hemorrhage }\end{array}$ \\
\hline 3 & 54 & $\mathrm{~F}$ & Left & Frontal & No & 68 & 3.5 & 3 & 15 & Yes & No & Glioblastoma & $\begin{array}{l}\text { Intracerebral } \\
\text { hemorrhage }\end{array}$ \\
\hline 4 & 46 & $\mathrm{M}$ & Right & $\begin{array}{l}\text { Fronto- } \\
\text { temporal }\end{array}$ & No & 37.1 & 18 & 8.6 & 10 & No & & Glioblastoma & \\
\hline 5 & 46 & $\mathrm{M}$ & Left & $\begin{array}{l}\text { Temporal- } \\
\text { Parietal }\end{array}$ & No & 51.2 & 8.3 & 11.4 & 9.8 & No & & Atypical Meningioma & \\
\hline 6 & 70 & M & Left & $\begin{array}{l}\text { Fronto- } \\
\text { temporal }\end{array}$ & No & 50.3 & 22 & 26.8 & 26.8 & No & & Meningioma & \\
\hline 7 & 36 & $\mathrm{~F}$ & Left & Temporal & No & 40 & 25.7 & 20.8 & 13 & No & & $\begin{array}{l}\text { Anaplastic } \\
\text { Astrocytoma }\end{array}$ & \\
\hline 8 & 56 & $\mathrm{M}$ & Left & Parietal & Yes & 37.7 & 0 & 9.6 & 10 & No & & Reactive Changes & \\
\hline 9 & 55 & M & Left & Parietal & Yes & 52.3 & 34.5 & 10.7 & 13.9 & No & & Reactive Changes & \\
\hline 10 & 55 & $\mathrm{~F}$ & Left & Temporal & No & 52.6 & 15.9 & 16.4 & 15.9 & No & & Glioblastoma & \\
\hline 11 & 74 & M & Right & Temporal & No & 34.3 & 36.2 & 28.2 & 28.2 & No & & Glioblastoma & \\
\hline 12 & 64 & $\mathrm{M}$ & Bilateral & Bicoronal & No & 48.8 & 70.1 & 44.5 & 83.4 & Yes & No & Atypical Meningioma & $\begin{array}{l}\text { Intracerebral } \\
\text { hemorrhage }\end{array}$ \\
\hline 13 & 57 & M & Left & Frontal & Yes & 69.2 & 21.2 & 33.3 & 35.8 & No & & Glioblastoma & \\
\hline 14 & 63 & $\mathrm{~F}$ & Left & Temporal & No & 22.3 & 30.5 & 20.3 & 16.4 & No & & $\begin{array}{l}\text { Anaplastic } \\
\text { Astrocytoma }\end{array}$ & \\
\hline 15 & 61 & M & Left & Parietal & No & 76.1 & 0 & 0 & 0 & No & & Glioblastoma & \\
\hline 16 & 69 & M & Right & Suboccipital & No & 49.2 & 10.6 & 60.9 & 53.4 & No & & $\begin{array}{l}\text { Metastatic } \\
\text { Adenocarcinoma }\end{array}$ & \\
\hline 17 & 55 & M & Bilateral & Bicoronal & No & 91.5 & 17.9 & 17.2 & 22.3 & No & & Metastatic Melanoma & \\
\hline 18 & 53 & $\mathrm{~F}$ & Left & $\begin{array}{l}\text { Fronto- } \\
\text { temporal }\end{array}$ & No & 41.7 & 30.1 & 40.5 & 52.3 & No & & Meningioma & \\
\hline 19 & 48 & M & Left & Temporal & No & 40.3 & 36.1 & 36.6 & 26.4 & No & & Glioblastoma & \\
\hline 20 & 52 & M & Right & Temporal & Yes & 12.8 & 37 & 27.8 & 14.6 & No & & Glioblastoma & \\
\hline 21 & 71 & $\mathrm{~F}$ & Right & Frontal & No & 73.5 & 15.1 & 0 & 37.3 & No & & Glioblastoma & \\
\hline
\end{tabular}




\section{Cureus}

\begin{tabular}{|c|c|c|c|c|c|c|c|c|c|c|c|c|c|}
\hline 22 & 38 & M & Left & Parietal & Yes & 44.1 & 0 & 12.4 & 10.1 & No & & Astrocytoma & \\
\hline 23 & 54 & M & Right & Temporal & Yes & 32 & 0 & 11.2 & 0 & No & & $\begin{array}{l}\text { Diffuse Large B-Cell } \\
\text { Lymphoma }\end{array}$ & \\
\hline 24 & 48 & $\mathrm{~F}$ & Right & Temporal & No & 56.8 & 0 & 14.8 & 0 & Yes & No & $\begin{array}{l}\text { Anaplastic } \\
\text { Astrocytoma }\end{array}$ & \\
\hline 25 & 60 & $\mathrm{~F}$ & Left & Temporal & Yes & 40.3 & 18.5 & 30.4 & 16.6 & Yes & No & Gliosarcoma & \\
\hline 26 & 48 & $\mathrm{~F}$ & Left & $\begin{array}{l}\text { Fronto- } \\
\text { temporal }\end{array}$ & No & 31.5 & 19 & 40.9 & 52.1 & No & & Meningioma & \\
\hline 27 & 55 & $\mathrm{~F}$ & Right & $\begin{array}{l}\text { Fronto- } \\
\text { temporal }\end{array}$ & No & 16.9 & 13.3 & 16.9 & 18.7 & Yes & Yes & $\begin{array}{l}\text { Metastatic } \\
\text { Adenocarcinoma }\end{array}$ & \\
\hline 28 & 56 & $\mathrm{M}$ & Right & Temporal & Yes & 31 & 14.5 & 43.2 & 11.7 & No & & Glioblastoma & \\
\hline 29 & 73 & $\mathrm{~F}$ & Left & Occipital & No & 26.1 & 51.6 & 22.6 & 23.4 & Yes & No & Glioblastoma & $\begin{array}{l}\text { Intracerebral } \\
\text { hemorrhage }\end{array}$ \\
\hline 30 & 57 & M & Left & Parietal & No & 49 & 17.2 & 13.1 & 18.7 & Yes & Yes & $\begin{array}{l}\text { Metastatic Small Cell } \\
\text { Carcinoma }\end{array}$ & \\
\hline 31 & 45 & $\mathrm{~F}$ & Right & Temporal & No & 44.3 & 42.7 & 17.8 & 32.5 & No & & Oligo & \\
\hline 32 & 54 & $\mathrm{M}$ & Right & Parietal & Yes & 35.7 & 36.7 & 22.6 & 10.9 & No & & Glioblastoma & \\
\hline 33 & 77 & $\mathrm{M}$ & Right & Temporal & No & 44.3 & 25 & 31 & 12.6 & No & & Glioblastoma & \\
\hline 34 & 55 & M & Right & Temporal & No & 38.5 & 36.7 & 35.7 & 0 & No & & $\begin{array}{l}\text { Anaplastic } \\
\text { Astrocytoma }\end{array}$ & \\
\hline 35 & 53 & $\mathrm{M}$ & Left & Frontal & Yes & 74.2 & 7.3 & 21.1 & 53.2 & No & & Glioblastoma & \\
\hline 36 & 63 & $\mathrm{~F}$ & Left & Frontal & No & 37.8 & 47 & 9.4 & 62.1 & No & & Meningioma & \\
\hline 37 & 42 & $\mathrm{M}$ & Right & Frontal & Yes & 34.3 & 31 & 6.3 & 21 & No & & Oligo & \\
\hline 38 & 71 & $\mathrm{M}$ & Left & Temporal & No & 50.6 & 26.4 & 36 & 10.7 & No & & Glioblastoma & \\
\hline 39 & 55 & M & Right & $\begin{array}{l}\text { Temporal- } \\
\text { Parietal }\end{array}$ & No & 19.1 & 43.9 & 31.9 & 32.2 & No & & Meningioma & \\
\hline 40 & 46 & $\mathrm{M}$ & Right & Occipital & Yes & 40.9 & 59.8 & 29.8 & 18.9 & No & & Glioblastoma & \\
\hline 41 & 27 & M & Right & Frontal & Yes & 71.4 & 0 & 0 & 0 & No & & Glioblastoma & \\
\hline 42 & 58 & $\mathrm{~F}$ & Left & Frontal & No & 31 & 41.4 & 15.7 & 24.8 & No & & $\begin{array}{l}\text { Metastatic Poorly } \\
\text { Differentiated } \\
\text { Carcinoma }\end{array}$ & \\
\hline 43 & 86 & $\mathrm{M}$ & Left & Occipital & No & 84.5 & 24.2 & 36.4 & 16.6 & Yes & No & Glioblastoma & \\
\hline 44 & 59 & $\mathrm{M}$ & Right & Parietal & No & 52.3 & 41.7 & 19.1 & 0 & No & & Glioblastoma & \\
\hline 45 & 60 & $\mathrm{~F}$ & Right & Occipital & Yes & 43 & 45.4 & 32.4 & 37.3 & No & & Glioblastoma & \\
\hline
\end{tabular}




\section{Cureus}

\section{TABLE 2: Patients Who Did Not Undergo Mapping}

WHO: World Health Organization

CST: Corticospinal Tract

SLF: Superior Longitudinal Fasciculus

AF: Arcuate Fasciculus

Oligo: Oligodendroglioma

MM: Millimeters

Pt: Patient

\section{With Monitoring}

Age

Mean (SD)

Median (Range)

$\operatorname{Sex}(\%)$

Male

Female

Laterality (\%)

Left

Right

Bicoronal

Revision (\%)

Speech Mapping (\%)

Motor Mapping (\%)

Both (\%)

Depth of Resection (mm)

Mean (SD)

Median (Range)

CST Distance (mm)

Mean (SD)
$51.75 \quad(17.78)$

$54 \quad(26-76)$

17

11

$(60.71 \%)$

$(39.29 \%)$

30

15

$(66.67 \%)$

$p=0.624$

13

$(46.43 \%)$

23

$(51.11 \%)$

$p=0.631$

15

(53.57\%)

20

(44.44\%)

0

$(0.00 \%)$

2

$(4.44 \%)$

$13 \quad(46.43 \%)$

15

(33.33\%)

$p=0.325$

$11 \quad(39.29 \%)$

$25 \quad(89.29 \%)$

8

(28.57\%)

45.91

(17.53)

$41.5 \quad$ (14.2-97)

45.94

(18.70)

$p=0.995$

43

(12.2-91.5)

$7.35 \quad(11.28)$

25.38

(17.03)

$p<0.0001^{*}$ 


\section{Cureus}

$\begin{array}{llllll}\text { Median (Range) } & 0 & (0-41) & 25 & (0-70.1) & \\ \text { SLF Distance (mm) } & & & & & \\ \text { Mean (SD) } & 8.44 & (10.49) & 21.83 & (13.84) & \mathrm{p}<0.0001^{*} \\ \text { Median (Range) } & 1 & (0-36.1) & 20.3 & (0-60.9) & \\ \text { Arcuate Fasciculus Distance (mm) } & & & & & \\ \text { Mean (SD) } & 11.31 & (12.03) & 21.86 & (18.46) & \mathrm{p}<0.004^{*} \\ \text { Median (Range) } & 10.9 & (0-42) & 16.6 & (0-83.4) & \\ \text { Deficit (\%) } & 7 & (25.00 \%) & 9 & (20.00 \%) & \mathrm{p}=0.772 \\ \text { Deficit Recovery (\%) } & 6 & (85.71 \%) & 2 & (22.22 \%) & \mathrm{p}=0.041^{*} \\ \text { GBM (\%) } & 17 & (60.71 \%) & 21 & (46.67 \%) & \mathrm{p}=0.336 \\ \text { Meningioma (\%) } & 0 & (0.00 \%) & 7 & (15.56 \%) & \\ \text { Metastasis (\%) } & 3 & (10.71 \%) & 6 & (13.33 \%) & \\ \text { Astrocytoma (\%) } & 3 & (10.71 \%) & 5 & (11.11 \%) & \\ \text { Oligodendroglioma (\%) } & 4 & (14.29 \%) & 2 & (4.44 \%) & \\ \text { Other (\%) } & 1 & (3.57 \%) & 4 & (8.89 \%) & \end{array}$

\section{TABLE 3: Comparative Statistics}

Student's unpaired t-test and Fisher's exact test were used to compare variables when appropriate.

* signifies statistically significant results, and include CST distance, SLF distance, arcuate fasciculus distance, and deficit recovery after surgery.

CST: corticospinal tract; GBM: glioblastoma; SD: standard deviation; SLF: superior longitudinal fasciculus

The clinical utility of AWBT is best illustrated through specific case examples. We present two patient vignettes that demonstrate the notable impact of AWBT on the ultimate treatment strategy, whether by affecting presurgical planning, intraoperative surgical decision making, and/or by helping localize white matter tracts.

\section{Case 1}

A 28-year-old female with a known history of anaplastic ependymoma (World Health Organization Grade III), which included two prior surgical resections and stereotactic radiosurgical treatment, presented with worsening left-hand paresis with loss of dexterity. An MRI of the brain with and without contrast demonstrated a 4-cm cystic lesion in the right frontal lobe with a focal nodule of enhancement at the medial border of the lesion (Figures $1 A-1 B)$. Calling on a priori knowledge of conventional sulcal anatomy, it was initially thought that this convexity lesion was displacing the corticospinal tracts (CSTs) posteriorly and medially. Since this was a superficial lesion, an anterior approach would be possible with 


\section{Cureus}

caution being taken when dissecting at the lateral and posterior margins.

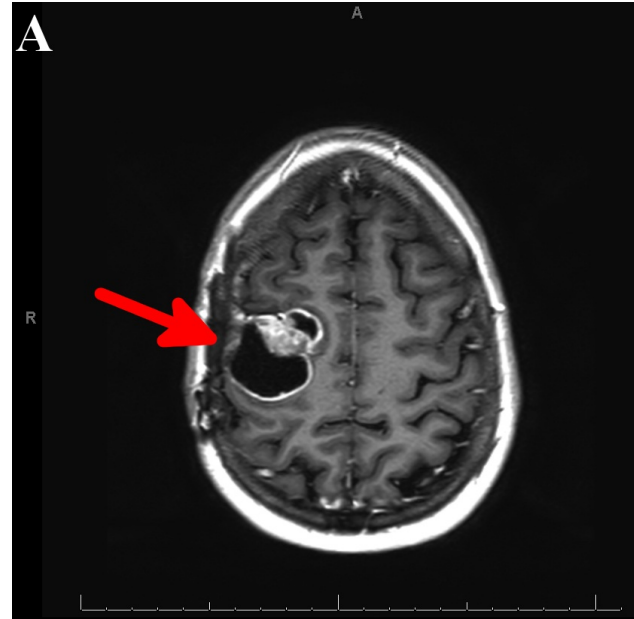

\section{B}

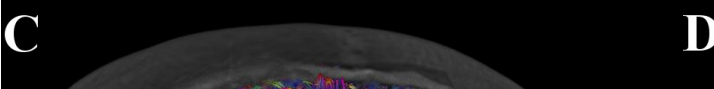

D

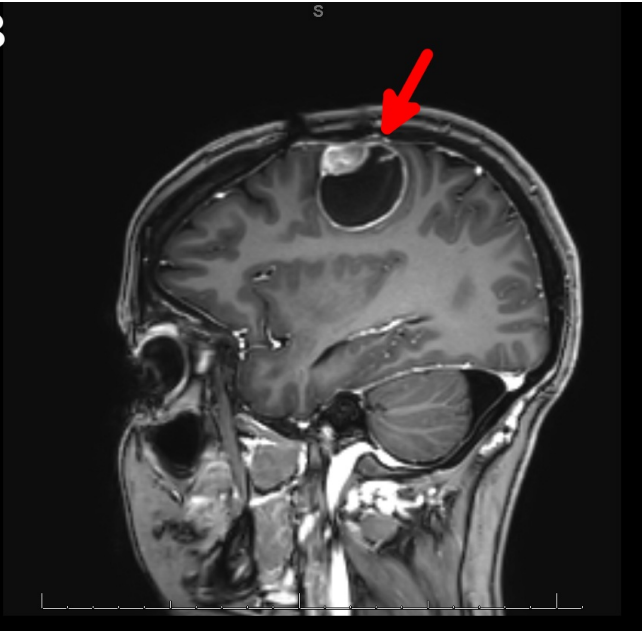

(1)
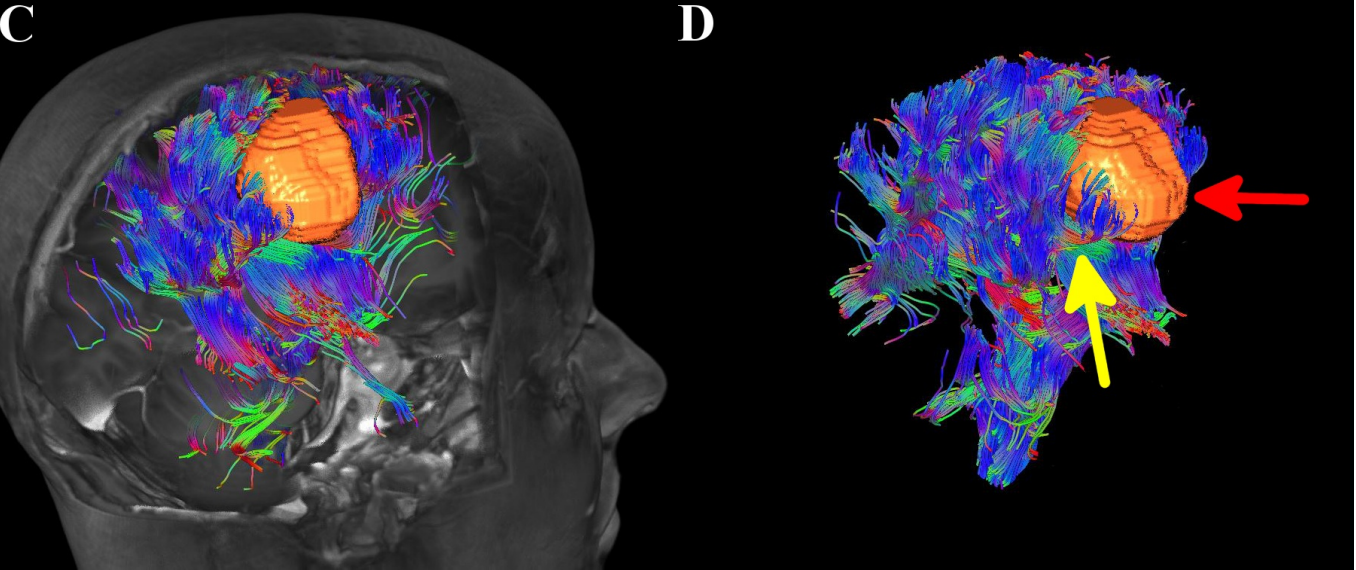

\section{FIGURE 1: MRI of the brain and automated whole brain tractography (AWBT) for Case 1}

A) Axial MRI of the brain with contrast showing a right-sided mass, denoted by a red arrow. B) Sagittal MRI of the brain with contrast showing a right-sided mass, denoted by a red arrow. C) AWBT image showing the tumor to be sitting within the corticospinal tract. D) AWBT image showing the tumor (denoted by red arrow), sitting within the corticospinal tract (denoted by yellow arrow).

AWBT: automated whole brain tractography; MRI: magnetic resonance imaging

AWBT revealed that the lesion sat within and also divided the CSTs (Figures $1 C-1 D$ ), and it was clear the previous surgical plan to enter the lesion anteriorly could jeopardize motor function. The surgical plan was modified whereby the lesion would be entered directly. The patient underwent an awake, right frontal craniotomy with intraoperative MRI and motor mapping, given that the AWBT demonstrated intimate proximity between the tumor and the CSTs. The reconstructed tractography data was fused with T1 MRI post-contrast imaging for stereotactic intraoperative neuronavigation. Cortical mapping was used to identify functional tissue as well as the boundaries of the lesion. Intraoperative mapping validated the tractography data - the lesion was embedded within the CST. Resection was undertaken in an en-bloc fashion with subsequent subcortical stimulation of the resection borders. In the deep posterior lateral border of the surgical cavity, left upper extremity motor responses were elicited consistently down to 4mAmp (Ojemann Cortical Stimulator, Integra Lifesciences, Plainsboro, NJ). This stimulation correlated well with tractography fused neuronavigation, again confirming the accuracy of 


\section{Cureus}

AWBT. Postoperatively, the patient had no change in her neurological examination, despite surgery between the fibers of the CST. A postoperative MRI confirmed a gross total resection of the lesion. Pathology again revealed anaplastic ependymoma. Her hospital course was uneventful and she was discharged home on postoperative day 3. Over long-term follow-up, the patient remained neurologically unchanged.

\section{Case 2}

A 44-year-old male with a known history of right parietal glioblastoma multiforme (World Health Organization Grade IV), which included post-subtotal resection, external beam radiation therapy, and adjuvant chemotherapy, presented to the clinic with an MRI demonstrating possible recurrence of disease (Figures $2 A-2 B$ ). AWBT showed that the tumor was lateral and adjacent to the CSTs, which allowed for surgery using a lateral approach (Figures 2C-2D). AWBT showed close proximity of the descending corticospinal tract (CST) to the lesion, and the decision was made for the patient to undergo a right parietal craniotomy with intraoperative neurophysiological monitoring using motor-evoked potentials and subcortical stimulation. This was paramount for safe removal of the tumor during the deep and medial portion of the resection.

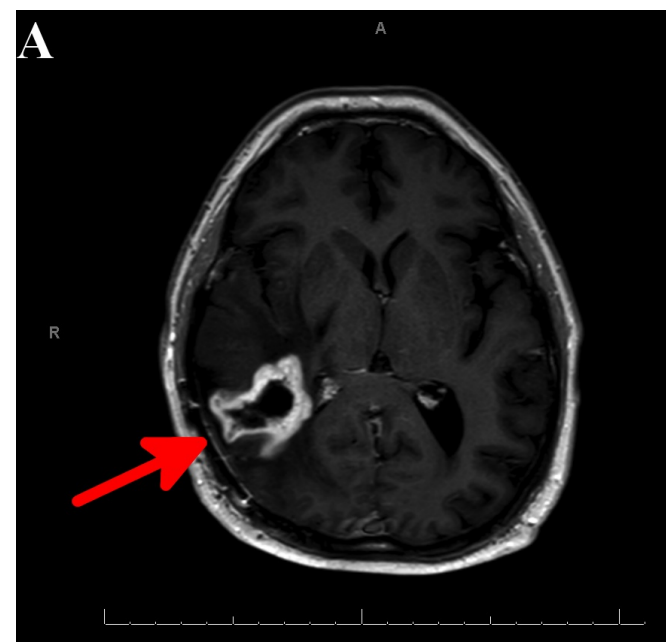

\section{B}

C

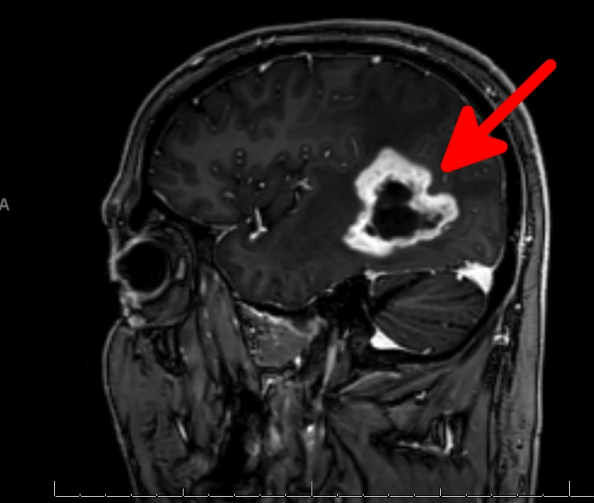

$\mathbf{D}$
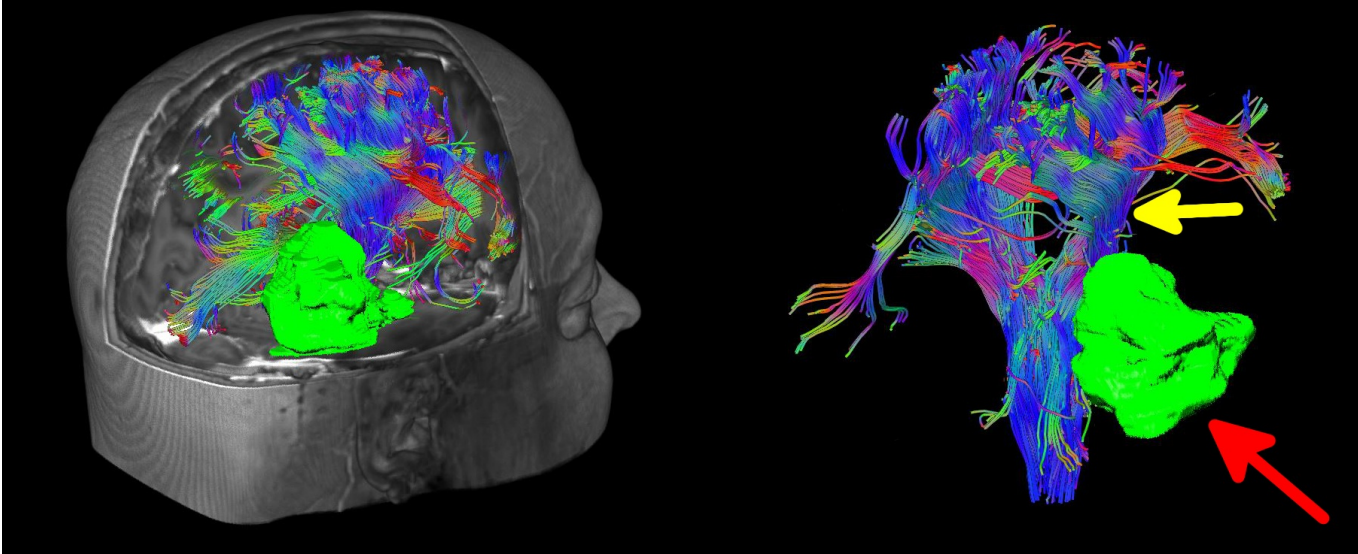

FIGURE 2: MRI of the brain and automated whole brain tractography(AWBT) for Case 2

A) Axial MRI of the brain with contrast showing a right-sided mass (denoted by red arrow). B) Sagittal MRI of the brain with contrast showing a right-sided mass (denoted by red arrow). C) AWBT image showing the tumor to be sitting deep and adjacent to the corticospinal tract. D) AWBT image showing the tumor (denoted by red arrow), sitting adjacent to the deep portions of the corticospinal 
The stereotactic wand fused with AWBT was used to determine the optimal corticectomy site, and the tumor was removed in a piecemeal fashion via an intralesional approach. Upon approaching the deep margin of the tumor, subcortical stimulation was correlated with AWBT to localize the CST. Continued careful resection was undertaken until normal-appearing tissue was encountered. AWBT neuronavigation confirmed that the CST was deep to the resection cavity. This correlated accurately with subcortical stimulation of $10 \mathrm{mAmp}$, implying a distance of at most $1 \mathrm{~cm}$ to the CST. Postoperatively, the patient maintained a stable neurological examination, with a postoperative MRI showing complete resection at the deep margins of the lesion adjacent to the CST. Pathology again showed glioblastoma multiforme. His hospital course was uneventful, and he was discharged home on postoperative day 2.

\section{Discussion}

The goals of neuro-oncologic surgery are cytoreduction, tissue sampling, and reduction of mass effect, all while preserving functional perilesional brain. Unfortunately, in neurological surgery, the separation between tumor and functional tissue can be difficult to ascertain. This is particularly true with essential white matter tracts, such as the corticospinal tract (responsible for motor function) and the arcuate fasciculus (essential for language) [2]. It is only recently that AWBT has come to fruition, allowing these tracts to be reliably and accurately identified without bias and within a clinically applicable timespan [6]. This accomplishment has overcome a historical workflow limitation and made individualized tractography relatively easy to implement.

In this study, we show that patients who were selected to undergo mapping had lesions closer to essential white matter tracts, including the corticospinal tract, superior longitudinal fasciculus, and the arcuate fasciculus. We also found that while patients who underwent mapping had the same risk of postoperative deficit as non-mapping patients, they were more likely to have postoperative recovery of their neurological deficit. This is a counter-intuitive result, given that even though this population of patients had lesions that were closer to eloquent white matter tracts (and thus considered high risk), they had a better overall outcome with mapping than patients with lesions at low risk for iatrogenic injury. While it may be argued that patients who require mapping for tumors are a different surgical population than patients who do not, we think comparisons between these two populations are appropriate. These two populations of patients are naturally separated into 'low-risk' and 'high-risk' groups for iatrogenic injury, with mapping as an extra precaution for patients who are high-risk (their lesions were significantly closer to eloquent white matter structures). Our institute also has a traditionally low threshold for mapping, implying that the non-mapping patients were very unlikely to have an injury. Despite this, both populations had the same rate of postoperative deficit, and the high-risk population had only a transient deficit. This helps to illustrate that while AWBT is a useful adjunct, it cannot replace the gold standard of mapping for high-risk lesions intraoperatively; however, AWBT can be used to help decide which patients are more appropriate for mapping, and thus contributing to increased postoperative recovery by identifying patients who have lesions near previously unseen essential white matter tracts.

While AWBT is a significant improvement to manual tractography, it is still subject to the limitations inherent in all tractography. Peritumoral edema disrupts the fractional anisotropy that DTI uses to clarify white matter tracts. Crossing fibers may be difficult to isolate due to their low anisotropy [9] and intraoperative brain shift may provide inaccurate localization data 
Given our strong caseload of examples where AWBT affected surgical decision making by localizing previously invisible white matter tracts, combined with how easy it is to obtain, AWBT is now considered for all patients who are undergoing intracerebral tumor resection. For lesions near eloquent brain structures, AWBT has become the standard of care at our hospital. While our findings agree with those of comparable single-center series [7-8], prospective studies are necessary to show how AWBT can improve clinical decision making to further support its use as a mainstay for surgery in eloquent areas.

\section{Conclusions}

While the current gold standard for localization of speech and language function remains direct cortical and subcortical stimulation, AWBT can provide critical preoperative and intraoperative information for the neurosurgeon. Our recent experience highlights the utility of AWBT in select cases of tumors near eloquent structures, and we provide specific examples of how AWBT changed the preoperative and intraoperative management of our surgical patients. Consequently, AWBT is now considered for all patients at our institution who are undergoing intracerebral tumor resection, regardless of whether we suspect the lesion to be near eloquent structures or not. Given the relative nascency of AWBT in tumor surgery, many questions regarding its role and applicability remain unanswered. Further studies are needed to determine the ideal patient population in whom to employ AWBT, what are the optimal tumor characteristics for AWBT, how peritumoral edema can affect AWBT accuracy, and does the supplemental use of AWBT have any effect on gross total resection or reoperation rates. The current body of evidence is largely predicated on case-control and cohort studies. In order to further delineate the role of AWBT in clinical practice, the neurosurgical community will benefit from prospective, randomized trials undertaken in well-defined patient populations.

\section{Additional Information}

\section{Disclosures}

Human subjects: Consent was obtained by all participants in this study. Henry Ford Hospital Insitutional Review Board issued approval 9755. Conflicts of interest: In compliance with the ICMJE uniform disclosure form, all authors declare the following: Payment/services info: All authors have declared that no financial support was received from any organization for the submitted work. Financial relationships: Ian Lee declare(s) personal fees from Medtronic. Ian Lee declare(s) personal fees from Monteris. Other relationships: All authors have declared that there are no other relationships or activities that could appear to have influenced the submitted work.

\section{Acknowledgements}

Sameah A Haider and Hesham M Zakaria contributed equally to this work. We thank Susan MacPhee for her editing.

\section{References}

1. Pillai JJ: The evolution of clinical functional imaging during the past 2 decades and its current impact on neurosurgical planning. AJNR Am J Neuroradiol. 2010, 31:219-25. 10.3174/ajnr.A1845

2. Essayed WI, Zhang F, Unadkat P, et al.: White matter tractography for neurosurgical planning: A topography-based review of the current state of the art. Neuroimage Clin. 2017, 15:659-72. 10.1016/j.nicl.2017.06.011

3. Villanueva-Meyer JE, Mabray MC, Cha S: Current clinical brain tumor imaging . Neurosurgery. 


\section{Cureus}

2017, 81:397-415. 10.1093/neuros/nyx103

4. O'Donnell LJ, Suter Y, Rigolo L, et al.: Automated white matter fiber tract identification in patients with brain tumors. Neuroimage Clin. 2017, 13:138-153. 10.1016/j.nicl.2016.11.023

5. Pujol S, Wells W, Pierpaoli C, et al.: The DTI challenge: toward standardized evaluation of diffusion tensor imaging tractography for neurosurgery. J Neuroimaging. 2015, 25:875-82. 10.1111/jon.12283

6. Christidi F, Karavasilis E, Samiotis K, et al.: Fiber tracking: A qualitative and quantitative comparison between four different software tools on the reconstruction of major white matter tracts. Eur J Radiol Open. 2016, 3:153-61. 10.1016/j.ejro.2016.06.002

7. D'Andrea G, Trillo G, Picotti V, Raco A: Functional magnetic resonance imaging (fMRI), preintraoperative tractography in neurosurgery: The experience of Sant' Andrea Rome University Hospital. Acta Neurochir Suppl. 2017, 124:241-50. 10.1007/978-3-319-39546-3_36

8. Celtikci E, Celtikci P, Fernandes-Cabral DT, Ucar M, Fernandez-Miranda JC, Borcek AO: Highdefinition fiber tractography in evaluation and surgical planning of thalamopeduncular pilocytic astrocytomas in pediatric population: Case series and review of literature. World Neurosurg. 2017, 98:463-69. 10.1016/j.wneu.2016.11.061

9. Assaf Y, Pasternak O: Diffusion tensor imaging (DTI)-based white matter mapping in brain research: a review. J Mol Neurosci. 2008, 34:51-61. 10.1007/s12031-007-0029-0

10. Javadi SA, Nabavi A, Giordano M, Faghihzadeh E, Samii A: Evaluation of diffusion tensor imaging-based tractography of the corticospinal tract: A correlative study with intraoperative magnetic resonance imaging and direct electrical subcortical stimulation. Neurosurgery. 2017, 80:287-99. 10.1227/NEU.0000000000001347 